\title{
New Insights into the Function of the Immunoproteasome in Immune and Nonimmune Cells
}

\author{
Hiroaki Kimura, ${ }^{1}$ Patrizio Caturegli, ${ }^{2}$ Masafumi Takahashi, ${ }^{1}$ and Koichi Suzuki ${ }^{3}$ \\ ${ }^{1}$ Division of Inflammation Research, Center for Molecular Medicine, Jichi Medical University School of Medicine, 3311-1 Yakushiji, \\ Shimotsuke, Tochigi 329-0498, Japan \\ ${ }^{2}$ Department of Pathology, The Johns Hopkins School of Medicine, Ross 656, 720 Rutland Avenue, Baltimore, MD 20205, USA \\ ${ }^{3}$ Department of Clinical Laboratory Science, Faculty of Medical Science, Teikyo University, 2-11-1 Kaga, Itabashi, \\ Tokyo 173-8605, Japan
}

Correspondence should be addressed to Hiroaki Kimura; balhiro33@jichi.ac.jp and Koichi Suzuki; koichis0923@med.teikyo-u.ac.jp

Received 10 July 2015; Accepted 10 September 2015

Academic Editor: Darren R. Flower

Copyright (C) 2015 Hiroaki Kimura et al. This is an open access article distributed under the Creative Commons Attribution License, which permits unrestricted use, distribution, and reproduction in any medium, provided the original work is properly cited.

\begin{abstract}
The immunoproteasome is a highly efficient proteolytic machinery derived from the constitutive proteasome and is abundantly expressed in immune cells. The immunoproteasome plays a critical role in the immune system because it degrades intracellular proteins, for example, those of viral origin, into small proteins. They are further digested into short peptides to be presented by major histocompatibility complex (MHC) class I molecules. In addition, the immunoproteasome influences inflammatory disease pathogenesis through its ability to regulate $\mathrm{T}$ cell polarization. The immunoproteasome is also expressed in nonimmune cell types during inflammation or neoplastic transformation, supporting a role in the pathogenesis of autoimmune diseases and neoplasms. Following the success of inhibitors of the constitutive proteasome, which is now an established treatment modality for multiple myeloma, compounds that selectively inhibit the immunoproteasome are currently under active investigation. This paper will review the functions of the immunoproteasome, highlighting areas where novel pharmacological treatments that regulate immunoproteasome activity could be developed.
\end{abstract}

\section{Introduction}

The immunoproteasome is a large proteolytic machine derived from the constitutive proteasome $[1,2]$ and plays a critical role in homeostasis and immunity. The constitutive proteasome is expressed ubiquitously in the body, where it degrades ubiquitinated proteins including transcriptional factors and proteins required for cell cycle progression $[3,4]$. Since the primary role of the immunoproteasome is to process antigens for presentation on major histocompatibility complex (MHC) class I molecules to $\mathrm{CD}^{+} \mathrm{T}$ lymphocytes [5], the immunoproteasome degrades various proteins, including viral proteins. Therefore, the immunoproteasome plays an important role during viral infection [6,7]. The expression of the immunoproteasome is induced by interferon- $\gamma$ (IFN$\gamma$ ) and tumor necrosis factor- $\alpha$ (TNF- $\alpha$ ) [8] under inflammatory conditions, such as infections, and autoimmune diseases when inflammatory cytokines are present [9]. Accordingly the immunoproteasome is controlled by factors that impact the immune system [10-13]. Interestingly, various roles for the immunoproteasome in nonimmune cells have been reported recently [14-16], suggesting that there could still be unknown roles for the immunoproteasome.

This review summarizes the roles of the immunoproteasome and recent efforts to develop novel therapeutic approaches by regulating immunoproteasome activity.

\section{Structure and Activity of the Immunoproteasome}

The immunoproteasome is a large proteolytic machinery derived from the constitutive proteasome (also known as the $26 \mathrm{~S}$ proteasome) and is expressed abundantly in immune cells, such as antigen-presenting cells [17-19]. The constitutive proteasome is expressed in the cytosol and nucleus of most cells, where it degrades ubiquitinated proteins to 
TABLE 1: Human immunoproteasome subunits.

\begin{tabular}{lcccc}
\hline Subunit & Proteolytic activity & Molecular weight & Chromosome & Alternative name \\
\hline $\mathrm{i} \beta 1$ & Chymotrypsin-like & $23.3 \mathrm{kD}$ & $6 \mathrm{p} 21.3$ & PSMB9, LMP2 \\
$\mathrm{i} \beta 2$ & Undefined & $28.9 \mathrm{kD}$ & $16 \mathrm{q} 22.1$ & PSMB10, LMP10, and MECL-1 \\
$\mathrm{i} \beta 5$ & Chymotrypsin-like & $30.4 \mathrm{kD}$ & $6 \mathrm{p} 21.3$ & PSMB8, LMP7 \\
PA28 $\alpha$ & N/A & $28.7 \mathrm{kD}$ & $14 \mathrm{q} 11.2$ & \\
PA28 $\beta$ & N/A & $27.4 \mathrm{kD}$ & $14 \mathrm{q} 11.2$ & \\
\hline
\end{tabular}

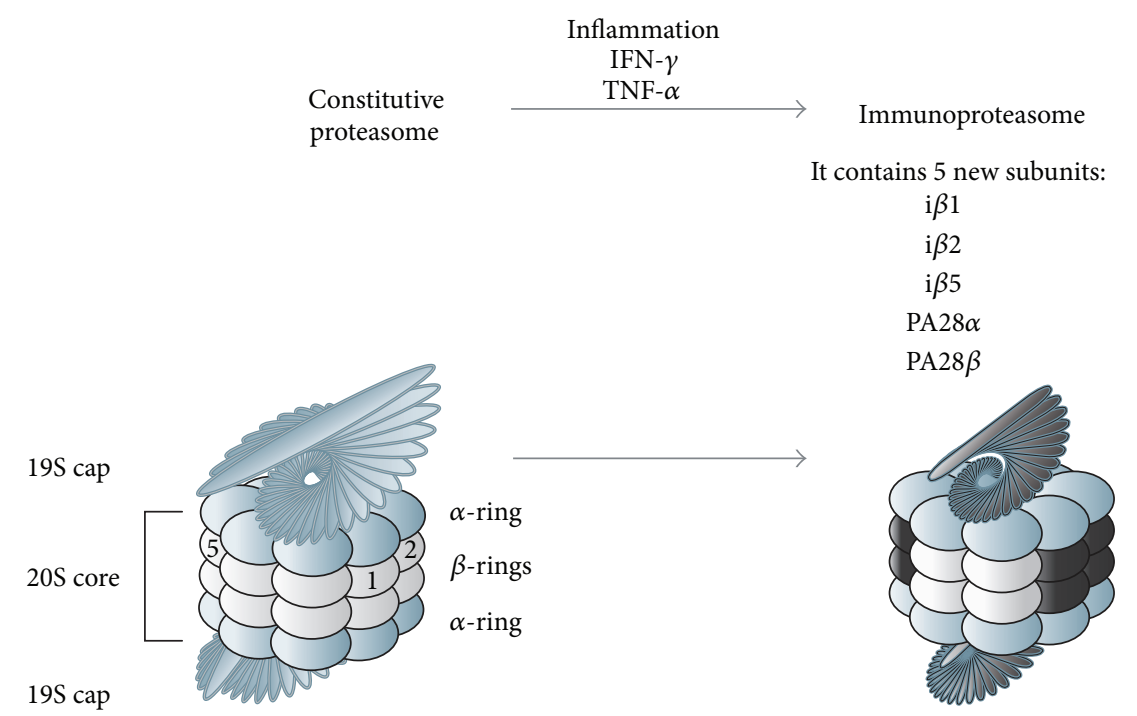

FIGURE 1: Structure of the constitutive proteasome and the immunoproteasome. The constitutive proteasome is composed of two pairs of inner $\beta$-rings, two pairs of outer $\alpha$-rings, and two caps (19S regulatory complexes). Inflammatory cytokines induce the expression of the five subunits (i $\beta 1$ [LMP2], i $\beta 2$ [LMP10], i $\beta 5$ [LMP7], PA $28 \alpha$, and PA28 $\beta$ ), which assemble on the proteasome core to create the immunoproteasome. When the induced subunits replace the $\beta$ subunits and 19S regulatory complex, the resulting multiprotein complex is called the immunoproteasome.

maintain cell viability and homeostasis $[4,20]$. For example, the constitutive proteasome degrades long-lived proteins, including proteins used for cell cycle progression and gene transcription. It is a large barrel-shaped protein complex $[21,22]$ composed of a catalytic $20 \mathrm{~S}$ core proteasome and two 19S regulatory complex components located at both ends of the $20 \mathrm{~S}$ core proteasome (Figure 1, left panel). The $20 \mathrm{~S}$ core proteasome has two pairs of outer $\alpha$ rings consisting of seven $\alpha$ subunits and two pairs of inner $\beta$ rings consisting of seven $\beta$ subunits. The three $\beta$ subunits $(\beta 1, \beta 2$, and $\beta 5)$ have proteolytic activities, including caspase-like activity for $\beta 1$, trypsin-like activity for $\beta 2$, and chymotrypsin-like activity for $\beta 5$ [23]. The $20 \mathrm{~S}$ core proteasome is usually capped at both ends by the $19 \mathrm{~S}$ regulatory complex [21, 22]. The $19 \mathrm{~S}$ regulatory complex recognizes ubiquitinated proteins and transfers them into the core of the proteasome where they are degraded by proteolysis.

When a cell is exposed to inflammatory stimuli, such as IFN- $\gamma$ and TNF- $\alpha$, five of the proteasome subunits are substituted with more efficient subunits: $\beta 1$ is replaced with $\mathrm{i} \beta 1$ (also known as large multifunctional peptidase 2 (LMP2) or proteasome subunit beta type 9 (PSMB9)), $\beta 2$ is replaced with $\mathrm{i} \beta 2$ (also known as LMP10, multicatalytic endopeptidase complex-like-1 (MECL-1), or PSMB10), $\beta 5$ is replaced with $\mathrm{i} \beta 5$ (also known as LMP7 or PSMB8), and the $19 \mathrm{~S}$ regulatory complex is replaced with the $11 \mathrm{~S}$ regulator composed of Proteasome Activator $\alpha$ (PA28 $\alpha)$ and PA28 $\beta$ (Figure 1, right panel and Table 1) [24-29]. This modified proteasome is called the immunoproteasome and it performs its proteolytic functions more efficiently than the constitutive proteasome [1]. For example, it degrades viral proteins for antigen presentation [7] and also processes ubiquitinated proteins, as does the constitutive proteasome [30]. Expression of the immunoproteasome subunits can be induced in nonimmune cells stimulated by IFN- $\gamma[13,16,31]$. Therefore, the immunoproteasome plays multiple roles, and the function of the immunoproteasome is not restricted to the immune system.

\section{Roles of the Immunoproteasome during Infection}

The best characterized role of the immunoproteasome is the processing of proteins in order to present antigenic peptides on MHC class I molecules (Figure 2) [32]. Deficiency of the immunoproteasome in mice reduces $\mathrm{CD}^{+} \mathrm{T}$ cell activation in hepatitis B virus (HBV) infection, lymphocytic choriomeningitis virus (LCMV) infection, and influenza virus infection $[7,33,34]$, although not in coxsackie virus B3 (CVB3) infection [35]. The immunoproteasome is also 


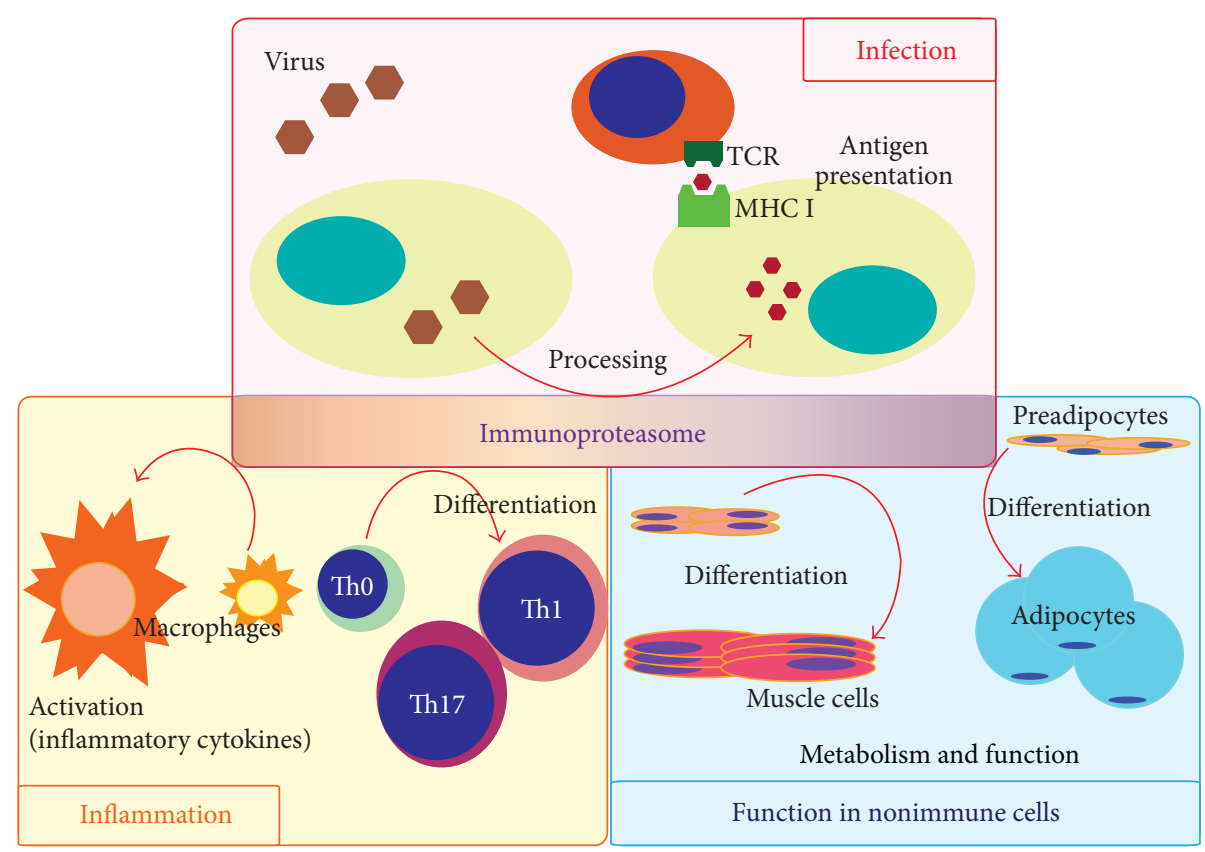

FIGURE 2: The immunoproteasome as a potential therapeutic target. The immunoproteasome plays an important role in immune responses, including processing viral proteins for antigen presentation, $\mathrm{T}$ cell differentiation, and macrophage activation. Recent studies have identified that the immunoproteasome is present in nonimmune cells, where it regulates cell differentiation and function.

important for activating the immune system against viral infection. For example, LMP2 deficiency reduced inflammatory cytokine (IL-1 $\beta$, IL-6, and TNF- $\alpha$ ) production during influenza viral infection [36]. Inflammatory cytokines, such as type I and type II IFNs and TNF- $\alpha$, induce the expression of the subunits that assemble into the immunoproteasome [37, 38]. Hepatitis C viral (HCV) infection or poly(I:C)-stimulation (mimicking viral infection) induces the expression of type I IFN (IFN- $\beta$ ) and the immunoproteasome subunits in hepatocytes [38]. Suppression of IFN$\beta$ inhibits expression of the immunoproteasome, and type I IFN (IFN- $\alpha$ ) treatment induces immunoproteasome expression in hepatocytes. Furthermore, Keller et al. showed that murine gammaherpesvirus-68 (MHV-68) infection induced expression of the immunoproteasome subunits in alveolar macrophages in the lung [16]. Thus, viral infection, IFN production, and expression of the immunoproteasome are strongly linked.

It should be noted that immunity against viral infections is not completely dependent on the immunoproteasome because there are some antiviral immune responses independent of the immunoproteasome [39]. In fact, mice lacking all of the immunoproteasome activities, generated by treating LMP2/LMP10 double-deficient mice with a LMP7-selective inhibitor, were still able to induce IFN- $\gamma$-producing cytotoxic $\mathrm{CD}^{+} \mathrm{T}$ cells upon LCMV infection [39].

\section{Roles of the Immunoproteasome in Inflammatory Diseases}

The immunoproteasome is involved in the pathogenesis of numerous inflammatory diseases, such as autoimmune diseases, by influencing $\mathrm{T}$ cell polarization, signaling through the nuclear factor- $\kappa \mathrm{B}(\mathrm{NF}-\kappa \mathrm{B})$ pathway, and the production of inflammatory cytokines by macrophage [40-45]. For example, Kalim et al. reported that LMP7 deficiency suppressed the differentiation of naive $\mathrm{CD} 4^{+} \mathrm{T}$ cells to Th1 and Th17 cells and instead promoted their differentiation to regulatory T cells (Figure 2) [46]. Maldonado et al. reported that deficiency of the immunoproteasome influenced NF$\kappa \mathrm{B}$ signaling [47]. The constitutive proteasome is involved in $\mathrm{NF}-\kappa \mathrm{B}$ signaling by degrading ubiquitinated I $-\kappa \mathrm{B}$. It remains to be defined how the constitutive proteasome and the immunoproteasome regulate NF- $\kappa$ B. Reis et al. reported that upregulated LMP7 expression in mouse macrophages due to LPS stimulation was suppressed by treatment with immunoproteasome inhibitors, including an LMP7 inhibitor (Figure 2) [48].

The immunoproteasome is essential for processing antigenic epitopes that are presented on MHC class I molecules to activate $\mathrm{CD}^{+} \mathrm{T}$ lymphocytes. The immunoproteasome is also involved in the regulation of NF- $\kappa \mathrm{B}$, which is essential for the transcription of many genes that encode inflammatory cytokines. Therefore, the activity of the immunoproteasome is essential in various inflammatory scenarios that result in pathological conditions. Thus, attempts were made to inhibit the immunoproteasome to identify potential treatments for inflammatory diseases. ONX-0914 (also known as PR-957) is a selective LMP7 inhibitor, which has been used as a treatment for autoimmune diseases in animal models. Muchamuel et al. reported that ONX-0914 attenuated experimental arthritis by blocking inflammatory cytokine expression [10]. As we mentioned, this LMP7 inhibitor blocked antigen presentation by MHC class I, suppressed 
the proliferation and activation of $\mathrm{CD} 8^{+} \mathrm{T}$ cells and Th17 cells, and lowered the production of inflammatory cytokines. The inhibitory effects probably contribute to the attenuation of disease progression in experimental arthritis.

Basler et al. showed that treatment with ONX-0914 significantly attenuated the clinical symptoms of experimental colitis and encephalomyelitis in mice [11, 12]. Expression of the immunoproteasome subunits (LMP2, LMP7, and LMP10) was upregulated in colitis lesions, which was induced in mice deficient in each of the immunoproteasome subunits. Colon lesions were significantly ameliorated in each of the deficient mouse strains compared to wild-type controls, and the amelioration was associated with suppressed inflammatory cytokine expression (TNF- $\alpha$, IL-1 $\beta$, IFN- $\gamma$, IL- 6 , IL-23, and IL17). Then, they examined the effect of ONX-0914 in experimental colitis and showed that treatment with ONX-0914 significantly improved colitis lesions. Although deficiency of the individual immunoproteasome subunits (i.e., LMP2, LMP7, or MECL-1) did not improve disease in a mouse model of experimental encephalomyelitis, treatment with ONX-0914 significantly attenuated disease progression and prevented a second exacerbation [12]. The authors mentioned that this discrepancy between immunoproteasome subunitdeficient mice and inhibitor-treated mice could be explained by the fact that endogenous chymotrypsin-like activity in monocytic cells contributes to pathogenesis and ONX-0914 inhibits chymotrypsin-like activity [12]. Deficiency of a single subunit is not able to suppress all chymotrypsin-like activity in the immunoproteasome because both LMP2 and LMP7 have chymotrypsin-like activity $[49,50]$. Overall, these studies suggest that ONX-0914 has potential for treating autoimmune diseases.

The immunoproteasome is involved in the pathogenesis of chronic thyroiditis [13]. Transgenic mice that express IFN- $\gamma$ specifically in the thyroid develop chronic thyroiditis and hypothyroidism [51, 52]. In this mouse model, LMP2 deficiency significantly improved inflammatory thyroid morphology and function [13]. Nagayama et al. reported that treatment with ONX-0914 improved Th1-type autoimmune thyroid disease (Hashimoto's thyroiditis), but not Th2-type autoimmune thyroid disease (Graves' disease), using mouse models [53]. Treatment with ONX-0914 suppressed IFN- $\gamma$ and IL-17 expression in the thyroid, which supports Basler's results.

LMP7 deficiency or treatment with ONX-0914 (a selective inhibitor of LMP7) seems to suppress inflammatory diseases with Th1 and Th17 cell-mediated inflammation. One report showed that LMP7 deficiency reduced Th2 responses in an asthma model [54]. LMP7 deficiency suppressed expression of the Th2 cytokines IL-4, IL-5, and IL-13 and infiltration of immune cells into the lung. The detailed mechanism of how LMP7 deficiency influences T cell polarization is still undefined. Because either Th1 or Th2 polarization is normally involved in the pathogenesis of many inflammatory diseases, it is necessary to know how the immunoproteasome influences $\mathrm{T}$ cell polarization in various inflammatory disease contexts in order to translate these findings to clinical studies.
TABLE 2: Human PSMB8 (LMP7 gene) alleles.

\begin{tabular}{lcc}
\hline Mutation & Influenced cytokines & Reference \\
\hline Thr 75 Met & IL-6, IL-8, and IFN- $\gamma$ & {$[58-60]$} \\
Cys 135 termination & Unknown & {$[60]$} \\
Gly 197 Val & IL-6 & {$[15]$} \\
Gly 201 Val & IL-6, IL-10 & {$[57]$} \\
\hline
\end{tabular}

\section{Roles of the Immunoproteasome in Nonimmune Cells}

Recent studies have examined the role of the immunoproteasome in nonimmune cells. Cui et al. reported that the immunoproteasome regulated skeletal muscle differentiation (Figure 2) [14]. They found that inhibiting the immunoproteasome by short hairpin RNA suppressed muscle differentiation using the mouse myoblast cell line $\mathrm{C} 2 \mathrm{C} 12$ and human skeletal muscle myoblasts. Proapoptotic proteins and apoptotic cells were upregulated by the treatment, which indicates that the immunoproteasome also regulates the degradation of proteins associated with apoptosis, as does the constitutive proteasome. They speculated that the immunoproteasome influences transcriptional factors associating with muscle differentiation. $\mathrm{Zu}$ et al. reported that the immunoproteasome regulated cardiac muscle mass in diabetic mice [55]. Streptozotocin (STZ) is commonly used to induce diabetic conditions in the experimental animal model. They showed that LMP2 expression was decreased in the hearts of STZ-injected mice. On the other hand, the expression of phosphatase and tensin homologue deleted on chromosome ten (PTEN) was upregulated, which impaired muscle regeneration [56]. LMP2 deficiency itself also leads to loss of cardiac muscle mass, which decreased cardiac function [55].

LMP7 has been associated with human disease, although no association has been found with the other immunoproteasome subunits (LMP2, LMP10, PA28 $\alpha$, and PA28 $\beta$ ). LMP7 mutation causes disease with autoinflammation and lipodystrophy [15, 57-59], and the number of cases is increasing $[60,61]$. As we described above, LMP7 plays a critical role in the immune system and is involved in cytokine expression. LMP7 mutation in humans causes abnormalities in cytokine expression, as listed in Table 2. Kitamura et al. showed that IL-6 expression was significantly higher in the skin lesions or sera of patients with LMP7 mutation [15], similar to other reports $[57,60]$. In particular, an association of LMP7 and lipodystrophy is interesting. Reduction of LMP7 expression by siRNA suppressed adipogenesis in 3T3L1 cells (Figure 2) [15]. LMP7 might be involved in lipid metabolic disorders because LMP7 is also associated with insulin-dependent diabetes mellitus [62], and inflammation is involved in the pathophysiology of metabolic diseases [63, 64]. To date, the role of the immunoproteasome in metabolic disorders and the endocrine system is poorly understood. We showed previously that overexpression of LMP2 was involved in the pathogenesis of chronic thyroid inflammation and hypothyroidism as described above [13]. In that study, we found that LMP2 was expressed in oxyphilic thyrocytes in 
humans and mice, and deletion of LMP2 in mice dramatically improved thyroid function and thyrocyte morphology [13]. These findings suggest an association between the immunoproteasome and endocrine metabolic function.

The lung is a vulnerable site for pathogens that induce chronic inflammation. Therefore, the immunoproteasome may play an important role in the lung. In fact, Keller et al. reported that immunoproteasome expression was detected in the lung parenchymal cells, for example, alveolar type I and II cells, fibroblasts, and bronchial epithelial cells at basal levels [16]. Viral infection and subsequent IFN secretion upregulated immunoproteasome expression in the lung. It is still not clear why those cells in the lung constitutively express LMP7 without infection or inflammation.

Considering the involvement of the immunoproteasome in cell differentiation and function, the immunoproteasome is important in nonimmune cells, too. Expression of the immunoproteasome in nonimmune cells during normal conditions has been found, although its role is not fully understood. Therefore, the role of the immunoproteasome in nonimmune cells should be addressed using mice deficient in the various immunoproteasome subunits, by knockdown of the immunoproteasome genes and with immunoproteasome inhibitors.

\section{Immunoproteasome Inhibitors and Their Clinical Relevance for Inflammatory Diseases and Neoplasms}

ONX-0914 is, thus far, the best characterized immunoproteasome inhibitor. As shown in the previous section, ONX0914 specifically inhibits LMP7 (i $\beta 5$ ), and it has been used in animal models and in vitro studies of inflammatory diseases $[10-12,46,53,65]$. Although selective inhibitors for LMP2 were not available when we reported that LMP2 deficiency suppressed thyroid inflammation and improved thyroid function [13], we expect that such inhibitors will be used to treat patients with chronic thyroiditis in the future. More studies are needed to analyze the mechanisms underlying the action of LMP2 on thyroid function.

Recently, immunoproteasome inhibitors have been investigated for application in clinical settings to treat hematopoietic neoplasms. Bortezomib is an inhibitor of $\beta 5$, a component of the constitutive proteasome, and has been used to treat multiple myeloma and mantle cell lymphoma [66]. Since the proteasome is responsible for the degradation of proteins involved in cell cycle progression, inhibition of proteasome function by bortezomib results in an accumulation of undigested proteins that leads to cell death.

Alternative treatments that overcome bortezomib-resistant malignancies have been characterized [66]. ONX-0912 is an inhibitor of both LMP7 (i $\beta 5)$ and $\beta 5$ and is effective for bortezomib-resistant myelomas [66, 67]. UK-101 and IPSI001 selectively inhibit LMP2 and exhibit antitumor activity against malignant myelomas $[68,69]$. Carfilzomib is effective for the treatment of myelomas and small cell lung cancers $[70,71]$. Proteasome subunits LMP7 (i $\beta 5$ ), LMP2 (i $\beta 1$ ), and $\beta 5$ have chymotrypsin-like activity. Since carfilzomib is a potent inhibitor of chymotrypsin-like activity [70], it appears likely that chymotrypsin-like activity is important for maintaining the proliferation of hematologic tumor cells. Precise differences in the chymotrypsin-like activity among the three subunits should be defined in order to understand how malignant cells acquire resistance to those proteasome inhibitors.

\section{Conclusion}

Regulating immunoproteasome expression and activity is a powerful tool for controlling cell function, which includes cell metabolism, differentiation, and immune regulation. So far, inhibitors of the immunoproteasome are widely available and applicable to the treatment of many inflammatory diseases and hematopoietic malignancies. In the near future, colitis and rheumatoid arthritis could be candidates for developing new treatments that target the immunoproteasome. In addition, metabolic diseases could provide additional candidates because the immunoproteasome is involved in both adipogenesis and inflammation of adipose tissue. As described in this review, most basic studies on the roles of the immunoproteasome in disease models have been achieved using mice (summarized in Figure 2). Since immunoproteasome enzymatic activity differs between species [72], findings from such basic studies should be carefully interpreted when considering the development of new therapeutic applications.

\section{Conflict of Interests}

The authors declare that there is no conflict of interests regarding the publication of this paper.

\section{Acknowledgments}

This work was supported by grants from the Japan Society for the Promotion of Science (JSPS) through the Grant-in-Aid for Scientific Research (C) and grants from Astellas Foundation for Research on Metabolic Disorders, Daiwa Securities Health Foundation, and Pancreas Research Foundation of Japan.

\section{References}

[1] K. Tanaka, "Role of proteasomes modified by interferon-gamma in antigen processing," Journal of Leukocyte Biology, vol. 56, no. 5, pp. 571-575, 1994.

[2] P.-M. Kloetzel and F. Ossendorp, "Proteasome and peptidase function in MHC-class-I-mediated antigen presentation," Current Opinion in Immunology, vol. 16, no. 1, pp. 76-81, 2004.

[3] K. Tanaka, "The proteasome: overview of structure and functions," Proceedings of the Japan Academy. Series B: Physical and Biological Sciences, vol. 85, no. 1, pp. 12-36, 2009.

[4] A. Ichihara and K. Tanaka, "Roles of proteasomes in cell growth," Molecular Biology Reports, vol. 21, no. 1, pp. 49-52, 1995.

[5] H. J. Fehling, W. Swat, C. Laplace et al., "MHC class I expression in mice lacking the proteasome subunit LMP-7," Science, vol. 265, no. 5176, pp. 1234-1237, 1994. 
[6] A. J. A. M. Sijts, S. Standera, R. E. M. Toes et al., "MHC class I antigen processing of an adenovirus CTL epitope is linked to the levels of immunoproteasomes in infected cells," The Journal of Immunology, vol. 164, no. 9, pp. 4500-4506, 2000.

[7] W. Chen, C. C. Norbury, Y. Cho, J. W. Yewdell, and J. R. Bennink, "Immunoproteasomes shape immunodominance hierarchies of antiviral $\mathrm{CD}^{+} \mathrm{T}$ cells at the levels of $\mathrm{T}$ cell repertoire and presentation of viral antigens," The Journal of Experimental Medicine, vol. 193, no. 11, pp. 1319-1326, 2001.

[8] P.-M. Kloetzel, "Antigen processing by the proteasome," Nature Reviews Molecular Cell Biology, vol. 2, no. 3, pp. 179-187, 2001.

[9] I. Shachar and N. Karin, "The dual roles of inflammatory cytokines and chemokines in the regulation of autoimmune diseases and their clinical implications," Journal of Leukocyte Biology, vol. 93, no. 1, pp. 51-61, 2013.

[10] T. Muchamuel, M. Basler, M. A. Aujay et al., "A selective inhibitor of the immunoproteasome subunit LMP7 blocks cytokine production and attenuates progression of experimental arthritis," Nature Medicine, vol. 15, no. 7, pp. 781-787, 2009.

[11] M. Basler, M. Dajee, C. Moll, M. Groettrup, and C. J. Kirk, "Prevention of experimental colitis by a selective inhibitor of the immunoproteasome," Journal of Immunology, vol. 185, no. 1, pp. 634-641, 2010.

[12] M. Basler, S. Mundt, T. Muchamuel et al., "Inhibition of the immunoproteasome ameliorates experimental autoimmune encephalomyelitis," EMBO Molecular Medicine, vol. 6, no. 2, pp. 226-238, 2014.

[13] H. J. Kimura, C. Y. Chen, S.-C. Tzou et al., "Immunoproteasome overexpression underlies the pathogenesis of thyroid oncocytes and primary hypothyroidism: studies in humans and mice," PLoS ONE, vol. 4, no. 11, article e7857, 2009.

[14] Z. Cui, S. M. Hwang, and A. V. Gomes, "Identification of the immunoproteasome as a novel regulator of skeletal muscle differentiation," Molecular and Cellular Biology, vol. 34, no. 1, pp. 96-109, 2014.

[15] A. Kitamura, Y. Maekawa, H. Uehara et al., "A mutation in the immunoproteasome subunit PSMB8 causes autoinflammation and lipodystrophy in humans," The Journal of Clinical Investigation, vol. 121, no. 10, pp. 4150-4160, 2011.

[16] I. E. Keller, O. Vosyka, S. Takenaka et al., "Regulation of immunoproteasome function in the lung," Scientific Reports, vol. 5, Article ID 10230, 2015.

[17] J. Haorah, D. Heilman, C. Diekmann et al., "Alcohol and HIV decrease proteasome and immunoproteasome function in macrophages: implications for impaired immune function during disease," Cellular Immunology, vol. 229, no. 2, pp. 139148, 2004.

[18] T. Frisan, V. Levitsky, and M. G. Masucci, "Variations in proteasome subunit composition and enzymatic activity in Blymphoma lines and normal B cells," International Journal of Cancer, vol. 88, no. 6, pp. 881-888, 2000.

[19] P. Leone, M. Di Tacchio, S. Berardi et al., "Dendritic cell maturation in HCV infection: altered regulation of MHC class i antigen processing-presenting machinery," Journal of Hepatology, vol. 61, no. 2, pp. 242-251, 2014.

[20] K. Tanaka, "Molecular biology of proteasomes," Molecular Biology Reports, vol. 21, no. 1, pp. 21-26, 1995.

[21] M. Unno, T. Mizushima, Y. Morimoto et al., "The structure of the mammalian 20 S proteasome at $2.75 \AA$ resolution," Structure, vol. 10, no. 5, pp. 609-618, 2002.
[22] M. Groll, L. Ditzel, J. Löwe et al., "Structure of 20S proteasome from yeast at $2.4 \AA$ resolution," Nature, vol. 386, no. 6624, pp. 463-471, 1997.

[23] G. N. DeMartino and C. A. Slaughter, "The proteasome, a novel protease regulated by multiple mechanisms," Journal of Biological Chemistry, vol. 274, no. 32, pp. 22123-22126, 1999.

[24] V. Ortiz-Navarrete, A. Seelig, M. Gernold, S. Frentzel, P. M. Kloetzel, and G. J. Hämmerling, "Subunit of the '20S' proteasome (multicatalytic proteinase) encoded by the major histocompatibility complex," Nature, vol. 353 , no. 6345, pp. 662664, 1991.

[25] R. Glynne, S. H. Powis, S. Beck, A. Kelly, L.-A. Kerr, and J. Trowsdale, "A proteasome-related gene between the two ABC transporter loci in the class II region of the human MHC," Nature, vol. 353, no. 6342, pp. 357-360, 1991.

[26] A. Kelly, S. H. Powis, R. Glynne, E. Radley, S. Beck, and J. Trowsdale, "Second proteasome-related gene in the human MHC class II region,” Nature, vol. 353, no. 6345, pp. 667-668, 1991.

[27] C. Realini, W. Dubiel, G. Pratt, K. Ferrell, and M. Rechsteiner, "Molecular cloning and expression of a $\gamma$-interferon-inducible activator of the multicatalytic protease," The Journal of Biological Chemistry, vol. 269, no. 32, pp. 20727-20732, 1994.

[28] J. Y. Ahn, N. Tanahashi, K. Akiyama et al., "Primary structures of two homologous subunits of PA28, a $\gamma$-interferon-inducible protein activator of the 20S proteasome," FEBS Letters, vol. 366, no. 1, pp. 37-42, 1995.

[29] N. Tanahashi, K.-Y. Yokota, J. Y. Ahn et al., "Molecular properties of the proteasome activator PA28 family proteins and $\gamma$ interferon regulation," Genes to Cells, vol. 2, no. 3, pp. 195-211, 1997.

[30] J. A. Nathan, V. Spinnenhirn, G. Schmidtke, M. Basler, M. Groettrup, and A. L. Goldberg, "Immuno- and constitutive proteasomes do not differ in their abilities to degrade ubiquitinated proteins," Cell, vol. 152, no. 5, pp. 1184-1194, 2013.

[31] M. E. Arellano-Garcia, K. Misuno, S. D. Tran, and S. Hu, "Interferon- $\gamma$ induces immunoproteasomes and the presentation of MHC I-associated peptides on human salivary gland cells," PLoS ONE, vol. 9, no. 8, Article ID e102878, 2014.

[32] P. M. Kloetzel, "Generation of major histocompatibility complex class I antigens: functional interplay between proteasomes and TPPII," Nature Immunology, vol. 5, no. 7, pp. 661-669, 2004.

[33] M. D. Robek, M. L. Garcia, B. S. Boyd, and F. V. Chisari, "Role of immunoproteasome catalytic subunits in the immune response to hepatitis B virus," Journal of Virology, vol. 81, no. 2, pp. 483491, 2007.

[34] E. Z. Kincaid, J. W. Che, I. York et al., "Mice completely lacking immunoproteasomes show major changes in antigen presentation," Nature Immunology, vol. 13, no. 2, pp. 129-135, 2012.

[35] E. Opitz, A. Koch, K. Klingel et al., "Impairment of immunoproteasome function by beta5i/lmp7 subunit deficiency results in severe enterovirus myocarditis," PLoS Pathogens, vol. 7, no. 9, Article ID e1002233, 2011.

[36] S. E. Hensley, D. Zanker, B. P. Dolan et al., "Unexpected role for the immunoproteasome subunit LMP2 in antiviral humoral and innate immune responses," Journal of Immunology, vol. 184, no. 8, pp. 4115-4122, 2010.

[37] F. Novelli and J.-L. Casanova, "The role of IL-12, IL-23 and IFN$\gamma$ in immunity to viruses," Cytokine and Growth Factor Reviews, vol. 15, no. 5, pp. 367-377, 2004. 
[38] E.-C. Shin, U. Seifert, T. Kato et al., "Virus-induced type I IFN stimulates generation of immunoproteasomes at the site of infection," The Journal of Clinical Investigation, vol. 116, no. 11, pp. 3006-3014, 2006.

[39] M. Basler, U. Beck, C. J. Kirk, and M. Groettrup, “The antiviral immune response in mice devoid of immunoproteasome activity," Journal of Immunology, vol. 187, no. 11, pp. 5548-5557, 2011.

[40] I. Gutcher and B. Becher, "APC-derived cytokines and T cell polarization in autoimmune inflammation," Journal of Clinical Investigation, vol. 117, no. 5, pp. 1119-1127, 2007.

[41] C. B. Schmidt-Weber, M. Akdis, and C. A. Akdis, "TH17 cells in the big picture of immunology," The Journal of Allergy and Clinical Immunology, vol. 120, no. 2, pp. 247-254, 2007.

[42] P. J. Barnes and M. Karin, "Nuclear factor- $\kappa \mathrm{B}-\mathrm{a}$ pivotal transcription factor in chronic inflammatory diseases," The New England Journal of Medicine, vol. 336, no. 15, pp. 1066-1071, 1997.

[43] P. P. Tak and G. S. Firestein, "NF-kappaB: a key role in inflammatory diseases," The Journal of Clinical Investigation, vol. 107, no. 1, pp. 7-11, 2001.

[44] P. Libby, "Inflammation in atherosclerosis," Arteriosclerosis, Thrombosis, and Vascular Biology, vol. 32, no. 9, pp. 2045-2051, 2012.

[45] J.-L. Davignon, M. Hayder, M. Baron et al., "Targeting monocytes/macrophages in the treatment of rheumatoid arthritis," Rheumatology, vol. 52, no. 4, Article ID kes304, pp. 590-598, 2013.

[46] K. W. Kalim, M. Basler, C. J. Kirk, and M. Groettrup, "Immunoproteasome subunit LMP7 deficiency and inhibition suppresses Th1 and Th17 but enhances regulatory $\mathrm{T}$ cell differentiation," Journal of Immunology, vol. 189, no. 8, pp. 4182-4193, 2012.

[47] M. Maldonado, R. J. Kapphahn, M. R. Terluk et al., "Immunoproteasome deficiency modifies the alternative pathway of NFkappaB signaling," PLoS ONE, vol. 8, no. 2, Article ID e56187, 2013.

[48] J. Reis, X. Q. Guan, A. F. Kisselev et al., "LPS-induced formation of immunoproteasomes: TNF- $\alpha$ and nitric oxide production are regulated by altered composition of proteasome-active sites," Cell Biochemistry and Biophysics, vol. 60, no. 1-2, pp. 77-88, 2011.

[49] Y. K. Ho, P. Bargagna-Mohan, M. Wehenkel, R. Mohan, and K.-B. Kim, "LMP2-specific inhibitors: chemical genetic tools for proteasome biology," Chemistry and Biology, vol. 14, no. 4, pp. 419-430, 2007.

[50] J. Reidlinger, A. M. Pike, P. J. Savory, R. Z. Murray, and A. J. Rivett, "Catalytic properties of $26 \mathrm{~S}$ and $20 \mathrm{~S}$ proteasomes and radiolabeling of MB1, LMP7, and C7 subunits associated with trypsin-like and chymotrypsin-like activities," The Journal of Biological Chemistry, vol. 272, no. 40, pp. 24899-24905, 1997.

[51] P. Caturegli, M. Hejazi, K. Suzuki et al., "Hypothyroidism in transgenic mice expressing IFN-gamma in the thyroid," Proceedings of the National Academy of Sciences of the United States of America, vol. 97, no. 4, pp. 1719-1724, 2000.

[52] H. Kimura, M. Kimura, W. H. Westra, N. R. Rose, and P. Caturegli, "Increased thyroidal fat and goitrous hypothyroidism induced by interferon-gamma," International Journal of Experimental Pathology, vol. 86, no. 2, pp. 97-106, 2005.

[53] Y. Nagayama, M. Nakahara, M. Shimamura, I. Horie, K. Arima, and N. Abiru, "Prophylactic and therapeutic efficacies of a selective inhibitor of the immunoproteasome for Hashimoto's thyroiditis, but not for Graves' hyperthyroidism, in mice," Clinical \& Experimental Immunology, vol. 168, no. 3, pp. 268273, 2012.
[54] A. Volkov, S. Hagner, S. Löser et al., "Beta5i subunit deficiency of the immunoproteasome leads to reduced Th2 response in OVA induced acute asthma," PLoS ONE, vol. 8, no. 4, Article ID e60565, 2013.

[55] L. Zu, D. Bedja, K. Fox-Talbot et al., "Evidence for a role of immunoproteasomes in regulating cardiac muscle mass in diabetic mice," Journal of Molecular and Cellular Cardiology, vol. 49 , no. 1, pp. 5-15, 2010

[56] Z. Hu, H. Wang, I. H. Lee et al., "PTEN inhibition improves muscle regeneration in mice fed a high-fat diet," Diabetes, vol. 59, no. 6, pp. 1312-1320, 2010.

[57] K. Arima, A. Kinoshita, H. Mishima et al., "Proteasome assembly defect due to a proteasome subunit beta type 8 (PSMB8) mutation causes the autoinflammatory disorder, Nakajo-Nishimura syndrome," Proceedings of the National Academy of Sciences of the United States of America, vol. 108, no. 36, pp. 14914-14919, 2011.

[58] A. K. Agarwal, C. Xing, G. N. DeMartino et al., "PSMB8 encoding the beta5i proteasome subunit is mutated in joint contractures, muscle atrophy, microcytic anemia, and panniculitisinduced lipodystrophy syndrome," The American Journal of Human Genetics, vol. 87, no. 6, pp. 866-872, 2010.

[59] A. Garg, M. D. Hernandez, A. B. Sousa et al., "An autosomal recessive syndrome of joint contractures, muscular atrophy, microcytic anemia, and panniculitis-associated lipodystrophy," Journal of Clinical Endocrinology and Metabolism, vol. 95, no. 9, pp. E58-E63, 2010.

[60] Y. Liu, Y. Ramot, A. Torrelo et al., "Mutations in proteasome subunit $\beta$ type 8 cause chronic atypical neutrophilic dermatosis with lipodystrophy and elevated temperature with evidence of genetic and phenotypic heterogeneity," Arthritis and Rheumatism, vol. 64, no. 3, pp. 895-907, 2012.

[61] K. Kunimoto, A. Kimura, K. Uede et al., "A new infant case of nakajo-nishimura syndrome with a genetic mutation in the immunoproteasome subunit: an overlapping entity with JMP and CANDLE syndrome related to PSMB8 mutations," Dermatology, vol. 227, no. 1, pp. 26-30, 2013.

[62] G. Y. Deng, A. Muir, N. K. Maclaren, and J.-X. She, "Association of LMP2 and LMP7 genes within the major histocompatibility complex with insulin-dependent diabetes mellitus: population and family studies," The American Journal of Human Genetics, vol. 56, no. 2, pp. 528-534, 1995.

[63] N. Rasouli and P. A. Kern, "Adipocytokines and the metabolic complications of obesity," Journal of Clinical Endocrinology and Metabolism, vol. 93, no. 11, pp. s64-s73, 2008.

[64] H. Sell, C. Habich, and J. Eckel, "Adaptive immunity in obesity and insulin resistance," Nature Reviews Endocrinology, vol. 8, no. 12, pp. 709-716, 2012.

[65] M. Mishto, M. L. Raza, D. de Biase et al., "The immunoproteasome beta5i subunit is a key contributor to ictogenesis in a rat model of chronic epilepsy," Brain, Behavior, and Immunity, vol. 49, pp. 188-196, 2015.

[66] A. M. Ruschak, M. Slassi, L. E. Kay, and A. D. Schimmer, "Novel proteasome inhibitors to overcome bortezomib resistance," Journal of the National Cancer Institute, vol.103, no. 13, pp. 10071017, 2011.

[67] D. Chauhan, A. V. Singh, M. Aujay et al., "A novel orally active proteasome inhibitor ONX 0912 triggers in vitro and in vivo cytotoxicity in multiple myeloma," Blood, vol. 116, no. 23, pp. 4906-4915, 2010.

[68] M. Wehenkel, J.-O. Ban, Y.-K. Ho, K. C. Carmony, J. T. Hong, and K. B. Kim, "A selective inhibitor of the immunoproteasome 
subunit LMP2 induces apoptosis in PC-3 cells and suppresses tumour growth in nude mice," British Journal of Cancer, vol. 107, no. 1, pp. 53-62, 2012.

[69] D. J. Kuhn, S. A. Hunsucker, Q. Chen, P. M. Voorhees, M. Orlowski, and R. Z. Orlowski, "Targeted inhibition of the immunoproteasome is a potent strategy against models of multiple myeloma that overcomes resistance to conventional drugs and nonspecific proteasome inhibitors," Blood, vol. 113, no. 19, pp. 4667-4676, 2009.

[70] F. Parlati, S. J. Lee, M. Aujay et al., "Carfilzomib can induce tumor cell death through selective inhibition of the chymotrypsin-like activity of the proteasome," Blood, vol. 114, no. 16, pp. 3439-3447, 2009.

[71] A. F. Baker, N. T. Hanke, B. J. Sands, L. Carbajal, J. L. Anderl, and L. L. Garland, "Carfilzomib demonstrates broad anti-tumor activity in pre-clinical non-small cell and small cell lung cancer models," Journal of Experimental \& Clinical Cancer Research, vol. 33, article 111, 2014.

[72] M. Raule, F. Cerruti, and P. Cascio, "Comparative study of the biochemical properties of proteasomes in domestic animals," Veterinary Immunology and Immunopathology, vol. 166, no. 12, pp. 43-49, 2015. 


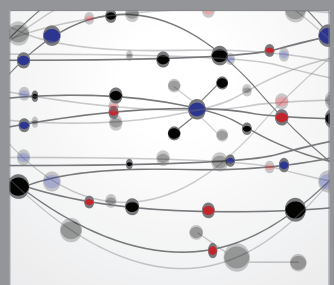

The Scientific World Journal
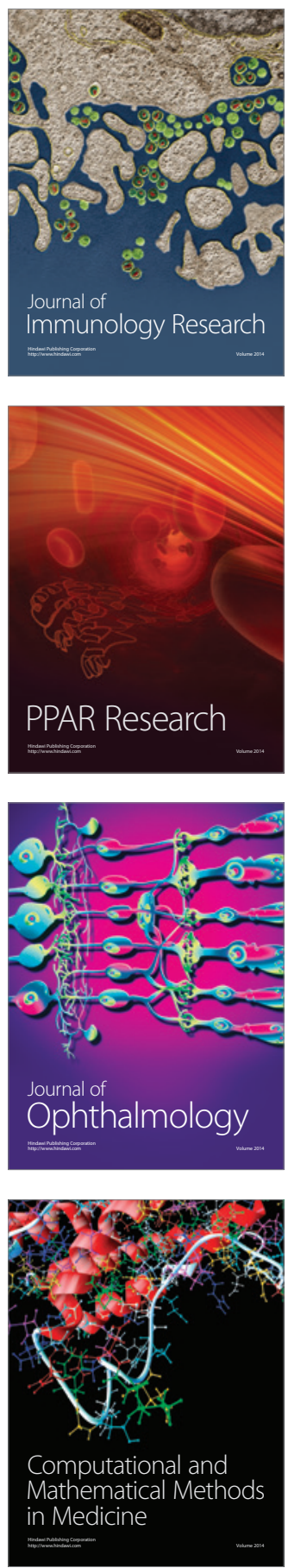

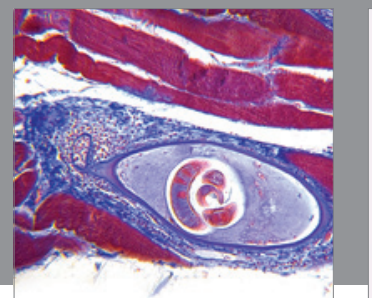

Gastroenterology

Research and Practice
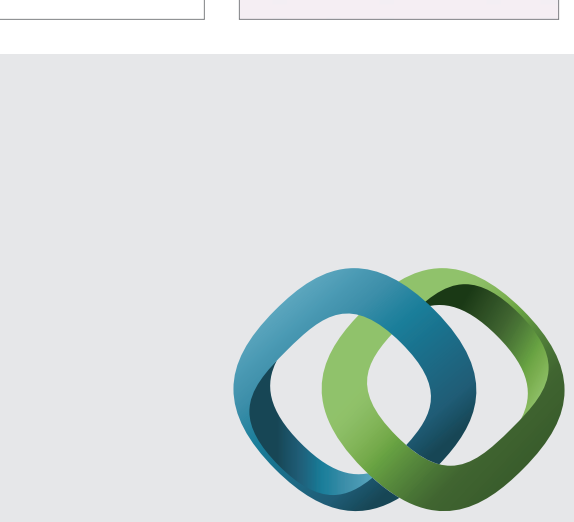

\section{Hindawi}

Submit your manuscripts at

http://www.hindawi.com
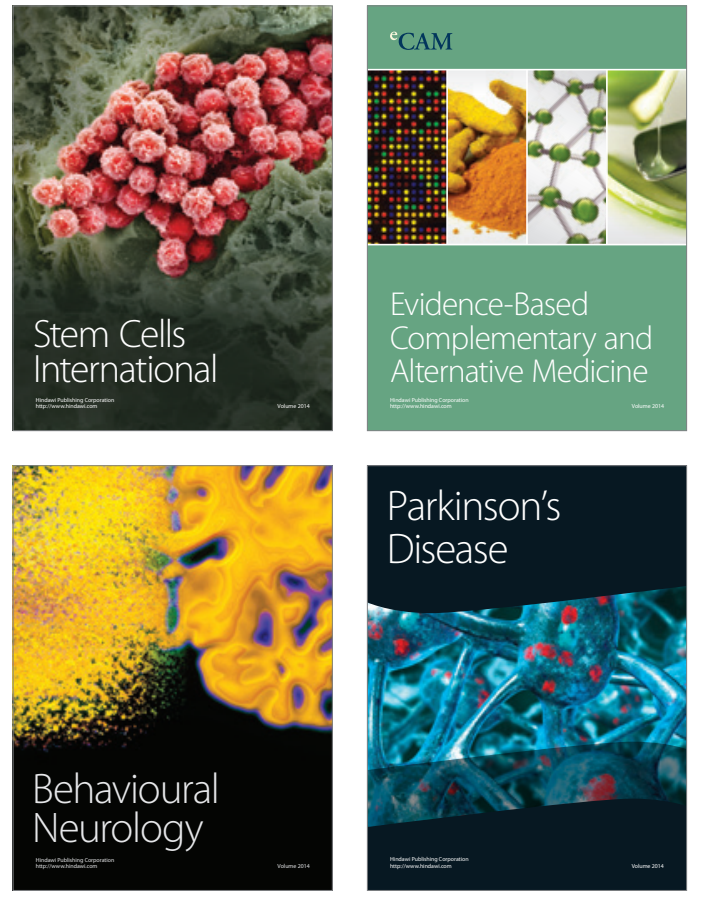
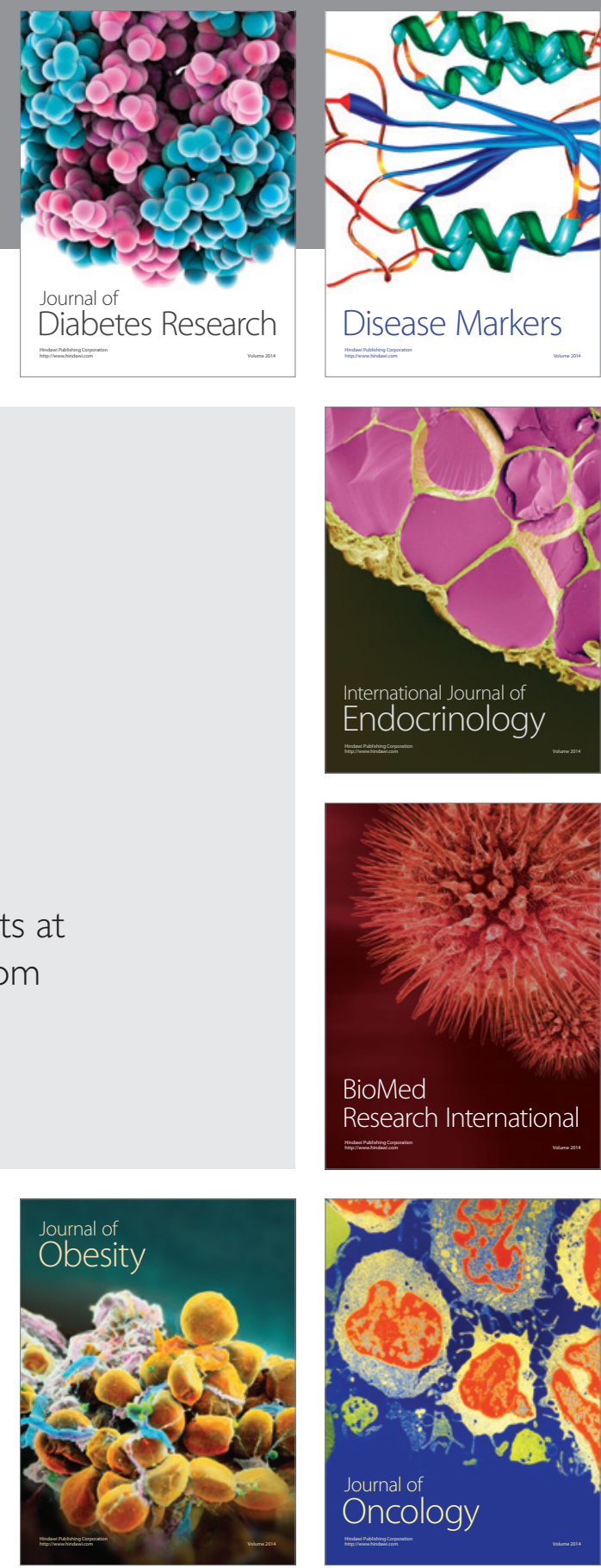

Disease Markers
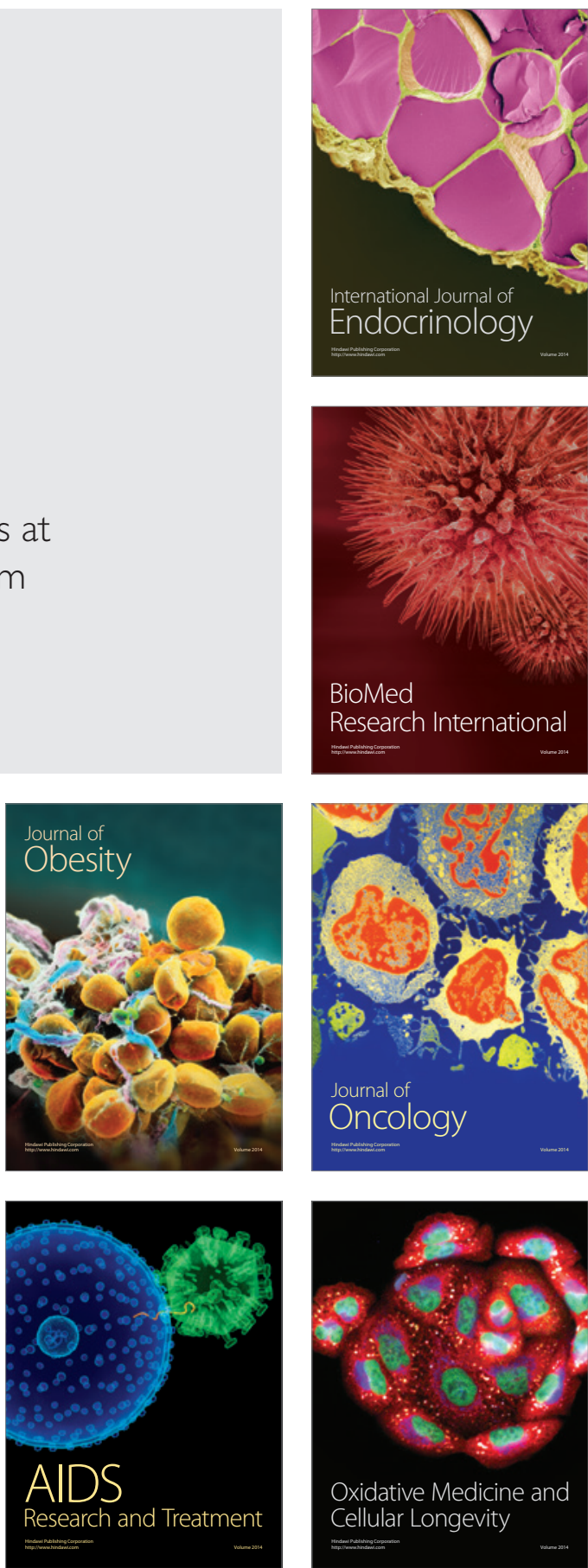